\title{
Efectos de la exposición a humo de tabaco sobre el asma bronquial en la infancia
}

\author{
PEDRO AGUILAR M.*
}

Effects of cigarette smoke exposure on child asthma

Cigarette smoking in children represents a major public health problem, all over the world. In South America, Chile occupies the first place in cigarette comsuption by children, even though many campaigns exist to avoid it. The devastating effect of tobacco exposure starts in fetal life and continues through lifetime. We present some main features in child asthma related to active and passive exposure to cigarette smoke.

Key words: Asthma, children, smoking.

\section{Resumen}

El consumo de tabaco en la población infantil representa un problema de salud pública significativo en diversas partes del mundo. En Sudamérica, Chile ocupa el primer lugar en consumo de tabaco infantil, pese a las campañas sanitarias implementadas para evitarlo. Los efectos dañinos del humo de tabaco, activo y pasivo, afectan al ser humano a lo largo de toda la vida, desde el período intrauterino. El objetivo del presente trabajo es revisar algunos tópicos referentes al efecto del tabaco sobre el asma bronquial en la niñez.

Palabras clave: Asma, niños, tabaco.

\section{Introducción}

El consumo de tabaco entre adultos y niños representa un problema de salud pública, tanto en la población general como entre aquellos con asma. La tendencia de la industria tabacalera mundial, durante los últimos años, se ha orientado a la incorporación de grupos vulnerables en dicho hábito, particularmente mujeres y niños ${ }^{1}$. La OMS estima en 1,25 billones el número de fumadores en el mundo, con cerca de 5 millones de muertes anuales asociadas a su consumo. De ellas, 15.000 se producen en Chile $^{2}$. El humo de tabaco representa un compleja mezcla de gases y partículas, con más de 4.000 componentes nocivos, no todos conocidos aún, entre los cuales destacan: nicotina, cuyo efecto sobre el sistema nervioso central da cuenta de la adicción observada entre fumadores; alquitranes, con marcados efectos irritantes y pro- inflamatorios sobre la vía aérea y el territorio alveolar; $\mathrm{CO}$, gas altamente afín a la hemoglobina, cuyo efecto determina deterioro en el transporte de oxígeno; y múltiples productos cancerígenos, como benceno, arsénico, cromo, entre otros $^{3}$. Estos productos emanan, como fase gaseosa y particulada, del tabaco en combustión, afectando al fumador activo como también, mediante la exposición en ambientes cerrados, a los fumadores pasivos, entre los cuales los niños representan un grupo altamente vulnerable. En el adulto, son ampliamente conocidas las asociaciones entre consumo activo y pasivo de tabaco con distintos tipos de cáncer, principalmente del sistema respiratorio alto y bajo, páncreas y vejiga; cardiopatía coronaria; enfermedad vascular periférica y EPOC ${ }^{3}$. En el niño, es un reconocido factor de riesgo de parto prematuro, bajo peso al nacer, Síndrome de Muerte Súbita en el lactante, morbilidad respiratoria alta * Departamento de Medicina Respiratoria Infantil, Hospital CRS El Pino. Escuela de Medicina, Universidad de
Santiago de Chile. 
y baja, hospitalizaciones por causa respiratoria, mayor prevalencia y severidad de asma y sibilancias $^{3-6}$. El objetivo de este artículo es revisar algunos tópicos relevantes referidos a la relación de tabaco y asma bronquial en la niñez.

\section{Prevalencia de tabaquismo en la población chilena}

La prevalencia de tabaquismo entre la población chilena adulta ha fluctuado entre 36,6 y $42,8 \% \%^{7-10}$. En la población infantil, la encuesta del Consejo Nacional para el Control de Estupefacientes (CONACE) de 1996 arrojó cifras de consumo de tabaco para el grupo de 12-18 años de $28,95 \%$ (mujeres $=31,2 \%$; hombres $=$ $27,04 \%$ ). Valdivia y colaboradores, en 2001 , comunican cifras preocupantes de tabaquismo entre escolares chilenos, con 5,3\% entre los 6-10 años, $31,1 \%$ entre $11-14$ años y $63,6 \%$ en $>14$ años $^{11}$. La prevalencia de consumo de tabaco el último mes, entre 2001 y 2005 , medido en el Sexto Estudio Nacional de Drogas en Población Escolar de Chile ( $8^{\circ}$ básico a $4^{\circ}$ medio, CONACE) demuestran cifras estables cercanas al $40 \%{ }^{12}$. En el "Primer estudio comparativo sobre uso de drogas en población escolar secundaria", realizado en Argentina, Bolivia, Brasil, Colombia, Chile, Ecuador, Paraguay, Perú y Uruguay (Septiembre 2006), las más altas prevalencias de consumo de tabaco se observaron, en todos los grupos etarios, entre estudiantes chilenos. La prevalencia por grupo etario fue: $\geq 17$ años: 54,83\%; 15-16 años: 44,81\%; < 14 años: $25,45 \%{ }^{13}$. Otro grupo vulnerable y expuesto precozmente al humo de tabaco lo representa el niño en gestación. Cifras extranjeras de tabaquismo durante el embarazo varían según edad, raza y educación entre $0-25 \%^{14,15}$. Pese a las campañas sanitarias realizadas en nuestro país, aún existe consumo de tabaco entre embarazadas. Recientemente, Mallol y colaboradores, comunicaron datos nacionales sobre tabaquismo durante el embarazo, en población de alto riesgo socioeconómico (Santiago-Sur) con una prevalencia global de $28 \%$, cifra que aumenta entre adolescentes embarazadas $(<19$ años) a un $46,7 \%^{16}$.

\section{Tabaquismo pasivo y asma}

Algunos reportes de tabaquismo pasivo, entre niños asmáticos, han fluctuado entre el $72,1 \%$ descrito en Zonguldak ${ }^{17}$ y el $28 \%$ en USA $^{18}$.
Halterman, comunica que cerca de la mitad de los niños con asma persistente convive con un fumador, de los cuales sólo el $64 \%$ tomará medidas destinadas a evitar el humo de tabaco en el hogar o en el automovil. Incluso, entre aquellos niños con asma severa, menos de $2 / 3$ fueron protegidos por los adultos con un ambiente libre de tabaco ${ }^{19}$. Al objetivar el grado de tabaquismo pasivo, Oddoze, encontró que el reporte de los padres de 79 niños expuestos a $0,1-10$ y $>10$ cigarros/día se asoció proporcional y significativamente a valores de cotinina en orina de 0,1 y $9,75 \mu \mathrm{g} / \mathrm{ml}$. Sin embargo, hubo diferencias significativas en el grado de exposición dependiendo de si era el padre o la madre quien fumaba, ya que cuando era sólo el padre (promedio 12,6 cigarros/día) el valor medio de cotinina fue de $3,5 \mu \mathrm{g} / \mathrm{ml}$, mientras que cuando era la madre (promedio 12 cigarros/día), el valor de cotinina urinaria subía a $20 \mu \mathrm{g} / \mathrm{ml}^{20}$. Lo anterior confirma la mayor exposición de los niños al humo de tabaco cuando la madre fuma, posiblemente dentro del hogar y en etapas de la vida con mayor cercanía del binomio madrehijo.

\section{Tabaquismo in útero y asma}

Existe cada día mayor información respecto de los efectos dañinos que produce el tabaquismo pasivo sobre un sujeto altamente vulnerable: el feto. Gilliland y colaboradores, en Carolina del Sur-USA, estudiando la asociación entre exposición in útero a tabaco y variables relacionadas con asma bronquial, encontraron riesgo aumentado, expresado como Odd Ratio e (IC) para: prevalencia de asma: $\mathrm{OR}=1,8(1,1-2,9)$; asma actual: $\mathrm{OR}=2,3(1,3-4)$; asma que requiere tratamiento el último año: $\mathrm{OR}=2,1(1,2-3,6)$; sibilancias asociadas a virus: $\mathrm{OR}=2,1 \quad(1,3-$ $3,4)$; sibilancias con ejercicio: $\mathrm{OR}=2,4(1,3-$ $4,3)$; crisis de asma: OR $=2,4(1,3-4,4)$; asma nocturna últimos 12 meses: $\mathrm{OR}=3,2(1,7-5,8)$; historia previa de sibilancias: $\mathrm{OR}=1,8(1,2-2,6)$; sibilancias persistentes: $\mathrm{OR}=3,1(1,6-6,1)$; consultas a emergencia el último año: $\mathrm{OR}=3,4$ $(1,4-7,8)^{15}$. En una cohorte de 4.089 recién nacidos seguidos por dos años, mediante cuestionarios dirigidos a los padres, se evaluó el efecto de la exposición al humo de cigarro, durante la vida fetal y postnatal temprana, sobre la salud respiratoria de los niños. Se aplicaron cuestionarios a los 2, 12 y 24 meses de edad. Cuando la madre fumó durante el embarazo y no durante el período postnatal, hubo un riesgo aumenta- 
do de presentar sibilancias recurrentes hasta los dos años de edad, con un OR (IC 95\%) de 2,2 $(1,3-3,6)$. Cuando hubo tabaco pasivo postnatal, con o sin tabaquismo materno in útero, el $\mathrm{OR}$ (IC 95\%) fue de 1,6 (1,2-2,3). También hubo mayor diagnóstico médico de asma, hasta los dos años, cuando existió tabaquismo in útero pero no cuando hubo exposición a tabaco postnatal, con un OR (IC 95\%) de 2,1 (1,2$3,7)^{21}$. En otra cohorte de madres, estudiadas en Odense y Aalborg (Dinamarca, 1984 a 1987), se siguió hasta 2002 un total de 11.144 niños empleando cuestionarios dirigidos a los padres. Mediante análisis de regresión múltiple, se evaluó en 7.844 niños la prevalencia de asma, rinitis, eczema y sibilancias antes de los 3 años. La exposición tardía in útero se asoció significativamente con sibilancias antes de los 3 años, con un OR (IC 95\%) de 1,2 (1,1-1,5), pero no a rinitis, eczema ni asma ${ }^{22}$. Rusconi, observó que los niños cuyas madres estuvieron expuestas a tabaco durante el embarazo tuvieron mayor riesgo de presentar sibilancias en todos los fenotipos de pacientes sibilantes con respecto al grupo control. Así, los OR (IC 95\%) para los fenotipos sibilantes transitorios tempranos, persistentes y sibilantes tardíos fueron $1,46(1,26-1,69), 1,81(1,5-2,19)$ y 1,18 $(0,99-1,42)$ respectivamente ${ }^{23}$. Mallol y colaboradores comunicaron, en nuestro país, mayor riesgo de Síndrome Bronquial Obstructivo (SBO), enfermedades respiratorias y hospitalizaciones por neumonía entre lactantes de alto riesgo socioeconómico cuando la madre fumaba en el embarazo. Sin embargo, al ajustar por tabaquismo actual, el mayor riesgo se mantuvo sólo para SBO, con un OR (IC) de 3,54 (1,17-10,7), confirmando lo observado en diversas publicaciones que relacionan, fundamentalmente, la exposición in útero al tabaco con una mayor prevalencia de sibilancias tempranas en la vida, más que la exposición postnatal a dicha noxa ${ }^{24}$.

\section{Tabaco, función pulmonar y clínica}

Es ampliamente conocida la asociación negativa entre exposición a humo de tabaco y función pulmonar, tanto en adultos como en niños. Dicho efecto se inicia precozmente. Stick y colaboradores, evaluaron la función pulmonar de 461 recién nacidos expuestos a tabaco in útero, antes de ponerse en contacto con tabaco ambiental ( $58 \mathrm{~h}$ de vida promedio). Las mediciones de tPTEF/tE (tiempo en alcanzar el flujo espiratorio máximo, a volumen corriente, versus el tiempo espiratorio, expresado como \%), con pletismografía de inductancia, revelaron menores valores en dicho índice entre hijos de madres que fumaron en el embarazo, reflejando un menor calibre de la vía aérea en etapas tempranas de la vida ${ }^{25}$. Mediante técnicas de compresión toracoabdominal rápida, Tepper ${ }^{26}$ y Hanraham $^{27}$, también describieron disminución de flujos espiratorios en la función pulmonar de lactantes expuestos in útero a tabaco, respecto de no expuestos. Estos y otros trabajos fundamentan la relación entre menor función pulmonar al nacer o durante los primeros meses con el mayor riesgo de presentar sibilancias los primeros años de la vida ${ }^{25}$. El efecto del tabaco in útero sobre la función pulmonar se mantiene en el largo plazo, como observó Gilliland en 3.357 escolares de California, USA, al comparar niños expuestos a tabaco in útero con no expuestos. Los primeros tuvieron menor función pulmonar en los índices: PEF (-3\%, IC95\% -4,4 a -1,4), FEMM $(-4,6 \%$, IC $95 \%-7$ a $-2,3), \mathrm{FEF}_{75}(-6,2 \%$, IC $95 \%-9,1$ a $-3,1)$, resultados que no se modifican al corregir por tabaco pasivo actual ${ }^{15}$. Se ha especulado que esta menor función pulmonar pudiera explicarse por algún grado de hipoplasia pulmonar, como por obstrucción bronquial periférica $^{28}$. Sin embargo, también pudiera relacionarse con una menor distensibilidad pulmonar total más que con cambios en la resistencia de la vía aérea ${ }^{29}$. Muchos son los efectos descritos de la nicotina sobre el pulmón fetal, capaces de explicar algunos de estos fenómenos. Cook y colaboradores, publicaron una revisión sistemática de trabajos que evaluaban la asociación de tabaco y reactividad bronquial en niños, encontrando evidencias de mayor reactividad bronquial entre niños expuestos a tabaco versus los no expuestos. En todos los trabajos analizados hubo mayor variabilidad del PEF cuando hubo exposición a tabaco ${ }^{30}$. Aguilar y cols, estudiaron la reactividad bronquial a metacolina entre escolares sanos, fumadores activos y no fumadores. Entre fumadores hubo hiperreactividad bronquial a metacolina $($ PC20 $<8 \mathrm{mg} / \mathrm{ml})$ en el $55 \%$ de los casos versus sólo el 19,2\% entre no fumadores, diferencias estadísticamente significativa ${ }^{31}$. Lo anterior sugiere una alteración funcional temprana de la vía aérea, sin expresión clínica, tal vez asociada a mecanismos adaptativos frente a esta noxa inhalada. En un metanálisis, Cook y colaboradores, demostraron un deterioro pequeño pero significativo de la función pulmonar $\left(\mathrm{VEF}_{1}\right)$ asociado a tabaquismo pasivo en niños, con un aparente mayor efecto de la exposición a tabaco in útero que a tabaco en la vida postnatal. 
Se ha sugerido que este déficit en la función pulmonar pudiera mantenerse en el tiempo, tal vez no como para explicar por sí solo las alteraciones funcionales de las enfermedades obstructivas del adulto, pero tal vez como coadyuvante del daño que determina el tabaco postnatal ${ }^{32}$. Moshammer y colaboradores, en un estudio multicéntrico que involucró a 9 países de Europa y Norte América, sobre un total de más de 20.000 niños entre 6 y 12 años, también encontraron menores valores espirométricos en niños expuestos a tabaco in útero $\left(\mathrm{VEF}_{1}-1 \%, \mathrm{FEM}_{25}\right.$ $-6 \%)$ y, en menor grado, cuando tuvieron exposición a tabaco pasivo postnatal ${ }^{33}$. Importa conocer el comportamiento en el tiempo de este deterioro, ya que se sabe, en adultos con EPOC, que la suspensión del tabaco disminuye la tasa de deterioro del $\mathrm{VEF}_{1}$ en el tiempo ${ }^{34,35}$. Muchas publicaciones han comunicado la asociación entre tabaquismo activo y pasivo sobre la salud respiratoria de los niños. En escolares de Carolina del Norte, la prevalencia de sibilancias los últimos 12 meses fue mayor cuando alguna vez fumaron activamente versus quienes nunca han fumado (23,9\% vs $14,1 \%$, respectivamente); cuando actualmente fuman la diferencia fue de $26 \%$ vs $15,9 \%$ y cuando están expuestos a tabaco pasivo versus controles, la diferencia fue de $20,3 \%$ vs $11,7 \%^{36}$. La exposición a tabaco en niños con asma se ha asociado a mayor número y severidad de las crisis, mayor uso de broncodilatadores de rescate y mayor tasa de hospitalizaciones por asma ${ }^{37}$. Evaluando la relación cuantitativa entre exposición a humo de tabaco y salud respiratoria en niños, Chilmonczyk, comunicó mayor número de exacerbaciones de asma por año, mientras mayor fuera la exposición a humo de tabaco. Se registraron $2,2 \pm 2$ crisis de asma/año entre niños no expuestos, $2,5 \pm 1,6$ crisis de asma/año si la madre u otro integrante de la familia fumaba y 3,9 $\pm 2,7$ crisis de asma/año si fumaba la madre y otro integrante del hogar. Al objetivar el grado de exposición mediante niveles de cotinina en orina, el número de crisis de asma por año fue: $2,1 \pm 1,9$ con niveles de cotinina urinaria $<10 \mathrm{ng} / \mathrm{ml} ; 2,8 \pm$ 1,68 con cotinina urinaria entre $10-39 \mathrm{ng} / \mathrm{ml} \mathrm{y}$ $3,6 \pm 2,9$ con cotinina urinaria $>39 \mathrm{ng} / \mathrm{ml}^{38}$.

\section{Tabaco: ¿Causa de asma?}

Una manera de cuantificar el efecto sobre la prevalencia de una condición clínica asociado a una noxa, es mediante el análisis de exceso de casos. Sturm estimó, entre niños de Carolina del Norte-USA, el exceso de casos con diagnóstico médico de asma determinado por la exposición a tabaco. Dicho exceso de casos fue de $14 \%$ cuando hubo exposición pasiva a tabaco y de $1 \%$ cuando era por tabaquismo activo, sumando un total de $15 \%$ de la prevalencia de asma atribuible a tabaco ${ }^{36}$. En nuestro país, Mallol y colaboradores encontraron, entre 4.738 adolescentes encuestados en el estudio ISAAC III, un exceso de casos con síntomas relacionados con asma, asociado al consumo de tabaco activo, igual a 223 niños, lo que representa más de un $27 \%$ de adolescentes con síntomas de asma atribuible al consumo de tabaco ${ }^{39}$. En un estudio epidemiológico basado en 14.578 adolescentes en Francia, Annesi-Maesano y cols, también encontraron incrementos en la prevalencia de asma y condiciones alérgicas, asociado al consumo activo de tabaco, luego de controlar por tabaquismo pasivo y otras variables. Entre los adolescentes expuestos a tabaco activo, hubo mayor riesgo de sibilancias durante el último año, asma alguna vez, rinoconjuntivitis alérgica actual y alguna vez, eczema actual y mayor severidad del asma. También hubo, entre fumadores pasivos versus no expuestos a tabaco, mayor riesgo de sibilancias el último año, rinoconjuntivitis alérgica y eczema actual ${ }^{40}$. Lo anterior podría estar relacionado con los hallazgos en modelos experimentales murinos, que han demostrado una activación exagerada de la respuesta Th2 en sujetos atópicos sometidos a pruebas de provocación bronquial y expuestos a humo de tabaco, comparado con aquellos no expuestos a este último ${ }^{41}$. Por otra parte, también en modelo animal (ratón) se ha demostrado que la coexistencia de estimulación antigénica, inductora de remodelación, con exposición crónica a humo de tabaco, determina mayores niveles de remodelación de la vía aérea, en particular engrosamiento del músculo liso, y aumento de la reactividad bronquial, mediado por aumento de quemokinas proinflamatorias tipo eotaxina- ${ }^{42}$. Por tanto, estudios funcionales y anatómicos en humanos y animales sugieren que fenómenos estructurales y bioquímicos pudieran explicar la mayor susceptibilidad a presentar enfermedades bronquiales obstructivas en la infancia cuando los niños se exponen tempranamemte a tabaco.

\section{Comentario}

Los datos alarmantes sobre consumo de tabaco en la infancia, sumado a la vasta información que lo relacionan con mayor prevalencia y 
severidad del asma bronquial, obligan a renovar esfuerzos para lograr ambientes libres de tabaco, para la población en general y para los niños en particular. Dado el conocimiento actual, modificar las tasas de tabaquismo materno podría incidir significativamente en la prevalencia del asma bronquial en la infancia.

\section{Bibliografía}

1.- ORGANIZACIÓN PANAMERICANA DE LA SALUD. Por una juventud sin tabaco: adquisición de habilidades para una vida saludable. Washington, D.C.: OPS, 2001. (Publicación Científica y Técnica No 579).

2.- Información sobre el Tabaco, MINSAL, www.minsal.cl.

3.- BELLO S, MICCHALLAND S, SOTO M, CONRERAS C, SALINAS J. Efectos de la exposición al humo de tabaco ambiental en no fumadores. Rev Chil Enf Respi 2005; 21: 179-92.

4.- HUNT C, HAUCK F. Sudden infant death syndrome. CMAJ 2006; 174: 1861-9.

5.- LUBIANCA J, HEMB L, BRUNELLI D. Systematic literature review of modifiable risk factors for recurrent acute otitis media in childhood. J Pediatria 2006; 82: 87-96.

6.- DI FRANZA J, ALIGNE C, WEITZMAN M. Prenatal and postnatal environmental tobacco smoke exposure and children's health. Pediatrics 2004; 113: 1007-15.

7.- BELLO S, MICHALLAND S, SOTO M, SALINAS J. Tabaquismo en funcionarios de educación. Rev Chil Enf Respir 2004; 20: 131-8.

8.- MINISTERIO DE SALUD. Encuesta Nacional de Salud-2003. Resumen Ejecutivo, Santiago.

9.- Consejo Nacional para el Control de Estupefacientes (CONACE). Ministerio del Interior. Gobierno de Chile. Quinto Estudio Nacional de Consumo de Drogas en Chile (2002), 2003.

10.- BELLO S, SOTO M, MICHALLAND S, SALINAS J. Encuesta nacional de tabaquismo en funcionarios de salud. Rev Méd Chile 2004; 132: 223-32.

11.- VALDIVIA G, SIMONETTI F, CUMSILlE P, RAMÍREZ V, HIDALGO C, PALMA B, et al. Consumo de tabaco en población menor de 18 años: estudio de prevalencia en escolares de Chile. Rev Méd Chile 2004; 132: 171-82.

12.- CONACE. Sexto Estudio Nacional de Drogas en Población Escolar de Chile, 2005. $8^{\circ}$ básico a $4^{\circ}$ medio. Informe final, Octubre de 2006.

13.- Oficina de las Naciones Unidas contra la Droga y el Delito (ONUDD) y otros. Sistema Subregional de Información e Investigación en Argentina, Bolivia, Chile, Ecuador, Perú y Uruguay, proyectos TDRLA-D74D75-D76-D77-D78, XLAI84.

14.- VENTURA S, HAMILTON B, MATHEWS T, CHANDRA A. Trends and variations in smoking during pregnancy and low birth weight: evidence from the birth certificate, 1990-2000. Pediatrics 2003; 111: 1176-80.

15.- GILLILAND F D, LI YU-FEN, PETERS J M. Effects of Maternal Smoking during Pregnancy and Environmental Tobacco Smoke on Asthma and Wheezing in Children. Am J Respir Crit Care Med 2001; 163: 429-36.

16.- MALLOL J, BRANDENBURG D, MADRID R,
SEMPERTEGUI F, RAMÍREZ L, JORQUERA D. Prevalencia de tabaquismo durante el embarazo en mujeres chilenas de bajo nivel socioeconómico. Rev Chil Enf Respir 2007; 23: 17-22.

17.- CENGIZLIER M, DIBEK E. Evaluation of risk factors in patients diagnosed as bronchial asthma. Allergol Immunopathol 2006; 34: 4-9.

18.- WEISS S T, HORNER A, SHAPIRO G, STENBERG A L. The prevelance of environmental exposure to perceived asthma triggers in children with mild-tomoderate asthma: data from the Childhood Asthma Management Program (CAMP). J Allergy Clin Immunol 2001; 107: 634-40.

19.- HALTERMAN J S, FAGNANO M, CONN K M, SZILAGYI P C. Do parents of urban children with persistent asthma ban smoking in their homes and cars? Ambul Pediatr 2006; 6: 115-9.

20.- ODOZZE C, DUBUS J C, BADIER M, THIRION X, PAULI A M, PASTOR J, et al. Urinary Cotinine and Exposure to Parental Smoking in a Population of Children with Asthma. Clinical Chemistry 1999; 45: 505-9.

21.- LANNERO E, WICKMAN M, PERSHAGEN G, NORDVALL L. Maternal smoking during pregnancy increases the risk of recurrent wheezing during the first years of life (BAMSE). Respir Res 2006; 5; 7: 3 .

22.- MAGNUSSON L L, OLESEN A B, WENNBORG H, OLSEN J. Wheezing, asthma, hayfever, and atopic eczema in childhood following exposure to tobacco smoke in fetal life. Clin Exp Allergy 2005; 35 (12): 1550-6.

23.- RUSCONI F, GALASSI C, CORBO G M, FORASTIERE F, BIGGERI A, CICCONE G, et al. Risk Factors for Early, Persistent, and Late-onset Wheezing in Young Children. Am J Respir Crit Care Med 1999; 160: 161722.

24.- MALLOL J, KOCH E, CARO N, SEMPERTEGUI F, MADRID R. Prevalencia de enfermedades respiratorias en el primer año de vida en hijos de madres que fumaron durante el embarazo. Rev Chil Enf Respir 2007; 23: 23-9.

25.- STICK S M, BURTON P R, GURRIN L, SLY P D, LE SOUEF $\mathrm{P}$ N. Effects of maternal smoking during pregnancy and a family history of asthma on respiratory function in newborns infants. Lancet 1996; 348: 1060-4.

26.- TEPPER R S, WILLIAMS-NKOMO T, MARTÍNEZ T, KISLING J, COATES C, DAGGY J. Parental smoking and airway reactivity in healthy infants. Am J Respir Crit Care Med 2005; 171: 78-82.

27.- HANRAHAN J P, TAGER I B, SEGAL M R, et al. The effect of maternal smoking in pregnancy on early infant lung function. Am Rev Respir Dis 1992; 145: 1129-35.

28.- BROWN R, HANRAHAN J, CASTILLE R, TAGER I. Effect of maternal smoking during pregnancy on passive respiratory mechanics in early infancy. Pediatr Pulmonol 1995; 19: 23-8.

29.- SEDDON P, DAVIS G, COATES A. Do tidal expiratory flow patterns reflect lung mechanics in infant?. Am J Respir Crit Care Med 1996; 153: 1248-52.

30.- COOK D G, STRACHAM D P. Parental smoking, bronchial reactivity and peak flow variability in children. Thorax 1998; 53: 295-301.

31.- AGUILAR P, OVALLE R, CORTEZ E, MALLOL J. Reactividad bronquial en escolares sanos fumadores y no fumadores. Rev Chil Enf Respir 1997; 13: 255.

32.- COOK D, STRACHAN D, CAREY I. Health effects 
of passive smoking 9: Parental smoking and spirometric indices in children. Thorax 1998; 53: 884-93.

33.- MOSHAMMER H, HOEK G, LUTTMANN-GIBSON H, NEUBERGER M A, ANTOVA T, GEHRING U, et al. Parental Smoking and Lung Function in Children. An International Study. Am J Respir Crit Care Med 2006; 173: 1255-63.

34.- WILLEMSE B W M, POSTMA D S, TIMENS W, TEN HACKEN N H T. The impact of smoking cessation on respiratory symptoms, lung function, airway hyperresponsiveness and inflammation. Eur Respir J 2004; 23: 464-76.

35.- SCANLON P D, CONNET J E, WALLER L A, ALTOSE M D, BAILEY W C, BUIST A S, et al. Smoking Cessation and Lung Function in Mild-toModerate Chronic Obstructive Pulmonary Disease. The Lung Health Study. Am J Respir Crit Care Med 2000; 161: 381-90.

36.- STURM J J, YEATTS K, LOOMIS D. Effects of Tobacco Smoke Exposure on Asthma Prevalence and Medical Care Use in North Carolina Middle School Children. Am J Public Health 2004; 94: 308-13.

37.- JAAKKOLA M S, JAAKKOLA J J K, BECKLAKE M R, ERNST P. Effect of passive smoking on the development of respiratory symptoms in young adults: an 8-year longitudinal study. J Clin Epidemiol 1996;
49: 581-6.

38.- CHILMONCZYK B A, SALMUN L M, MEGATHLIN K N, NEVEUX L M, PALOMAKI G E, KNIGHT G J, et al. Association between Exposure to Environmental Tobacco Smoke and Exacerbations of Asthma in Children. N Engl J Med 1993; 328: 1665-9.

39.- MALLOL J, CASTRO-RODRÍGUEZ J A, CORTEZ E. Effects of Active Tobacco Smoking on The Prevalence of Asthma-Like Symptoms in Adolescents. The I J COPD (en prensa).

40.- ANNESI-MAESANO I, ORYSZCZYN M P, RAHERISON C, KOPFERSCHMITT C, PAULI G, TAYTARDS A, et al. Increased prevalence of asthma and allied diseases among active adolescent tobacco smokers after controlling for passive smoking exposure. A cause for concern?. Clin Exp Allergy 2004; 34: 1017-23.

41.- SEYMOUR B, PINKERTON K, FRIEBERTSHAUSER K, COFFMAN R, GERSHWIN L. Second-hand smoke is an adjuvant for $\mathrm{T}$ helper-2 responses in a murine model of allergy. J Immunol 1997; 159: 6169-75.

42.- MIN M, SONG D, MILLER M, CHO J, MCELWAIN S, FERGUSON P, et al. Coexposure to Environmental Tobacco Smoke Increases Levels of Allergen-Induced Airway Remodeling in Mice. J Immunol 2007; 178: 5321-8.

Correspondencia a:

Dr. Pedro Aguilar Miranda

Av. Padre Hurtado 13560, San Bernardo

Santiago, Chile

E-mail: aguilarped@gmail.com 